\title{
La situation juridique de l'occupant sans titre d'une parcelle du domaine national au Cameroun
}

\author{
Par Prosper Nkou Mvondo
}

\section{Introduction}

Relativement au droit immobilier, la démarche législative suivie par l'Etat camerounais ${ }^{1}$ entre en rupture avec la distinction classique entre domaine public et domaine privé. Une catégorie de terre assez particulière a vu le jour avec la réforme foncière de 1974: le "domaine national", qui a remplacé la notion de "patrimoine collectif national" consacrée par le décret-loi du 9 janvier 1963.

Le domaine national est une catégorie résiduelle. Il s'agit de ces vastes étendues de terres qui forment le territoire camerounais et qui ne font partie, ni du domaine public, ni du domaine privé ${ }^{2}$. En vertu de l'Ordonnance de 1974, l'Etat est chargé de la gestion des ces terres $^{3}$. De grands espaces du domaine national sont libres de toute occupation (savanes inhabitées, forêts vierges...). D'autres, par contre, sont occupés par des particuliers. Parmi ceux-ci, il y en a qui ont des titres d'occupation délivrés par l'État gestionnaire (contrat de concession provisoire, permis d'occuper). Beaucoup de terres sont toutefois occupées par des particuliers qui n'ont pas jugé utile de s'adresser à l'Etat gestionnaire. Il s'agit alors d'occupants sans titre.

En zone urbaine, avec la pénurie des logements, les espaces aux alentours des centres villes sont pris d'assaut par des populations en mal de logement. Cette mainmise spontanée sur la terre permet à une grande masse de travailleurs et de petits salariés de se loger. Loin d'être éphémères, ces occupations du domaine national se transforment en installations définitives, aussi bien en réalité que dans la conscience des occupants.

Le Cameroun n'est pas seul dans ce cas. Le Sénégal, le Togo ont adopté la même démarche dans leurs réformes du droit foncier.

2 Le domaine privé peut être celui de l'Etat ou celui des particuliers. Dans tous les cas, un titre foncier est exigé. Ce document est le seul titre et mode de preuve de la propriété immobilière au Cameroun.

3

L'Etat n'est pas propriétaire de ces terres comme c'est la cas au Congo ex-Zaïre. 
En zone rurale, le domaine national est occupé et exploité par des populations, le plus souvent autochtones, se prévalant d'un droit de propriété coutumier qui est pourtant ignoré par la législation foncière en vigueur. La réforme de 1974 a pourtant donné l'opportunité à ces populations de devenir propriétaires de ces espaces en obtenant des titres fonciers. Mais celles-ci n'ont pour la plupart jamais trouvé la nécessité d'engager une quelconque procédure pour l'obtention de quelque titre foncier que ce soit, s'estimant légitimement propriétaires de ces terres qu'elles tiennent de leurs ancêtres ${ }^{4}$. Quoiqu'il en soit, au regard de la législation foncière, ces populations sont de simples occupants de fait, c'est-à-dire sans titre. Sur le plan du droit, la situation juridique de la plupart de ceux qui occupent ainsi sans titre le domaine nationale, peut s'analyser en terme de possession ${ }^{5}$. Pour que l'on parle de possession, il faut que soient réunies deux composantes: le corpus et l'animus. Le corpus est l'élément objectif de la possession. C'est l'exercice de fait des prérogatives correspondant à un droit. Puisqu'il s'agit souvent du droit de la propriété, le corpus est le fait de se comporter comme un propriétaire, en exerçant sur la chose: l'usus, le fructus et l'abusus.

Les occupants sans titre des parcelles du domaine national se comportent généralement en propriétaire des lieux. Ils s'installent, usent du sol, tirent des fruits de l'emprise qu'ils ont sur la terre ; ils récoltent, reçoivent des payements de loyers sur les maisons construites sur le domaine national... On retrouve là des éléments du droit de propriété: l'usus et le fructus. On retrouve aussi l'abusus à travers les ventes de ces terres par lesdits occupants.

Seulement, on peut accomplir tous ces actes matériels sur un bien sans prétendre en être propriétaire. On ne saurait alors parler de possession. Ce qui n'est pas le cas des occupants du domaine national. Ces derniers sont le plus souvent convaincus d'être propriétaires desdites parcelles. Le phénomène des résistances à la législation foncière officielle sont là

Il faut dire que le législateur colonial avait encouragé cette attitude des populations en consacrant le caractère facultatif de l'immatriculation pour les indigènes (décret du 21 juillet 1932, article 2, décret du 26 juillet 1932, article 5). Selon Solus, le législateur colonial français ne voulait pas semble-t-il, "imposer aux indigènes un régime foncier qui n'est pas sans altérer leurs institutions coutumières" (Solus, Traité de la condition des indigènes, Paris, Recueil Sirey, 1927, n³50). Voir aussi, Demaison, Le régime de la propriété foncière en AEF, Revue Juridique et Politique de $l^{\prime}$ Union Française, $\mathrm{n}^{\circ} 3,1956$, page 426. On pourrait donc expliquer cette attitude des populations aujourd'hui par les réminiscences de cette pensée liée à la politique législative de l'époque. Le législateur post-colonial et surtout celui de 1974, a adopté une solution inverse en encourageant l'accès à la propriété. Pourtant, les populations ne s'activent pas beaucoup pour immatriculer les terres qu'elles occupent ou exploitent. Peut-être que les difficultés pratiques que ces populations rencontrent face à l'administration y sont pour quelque chose (gratuité chimérique, lenteurs, corruption des fonctionnaires...).

Voir A. Minkoa She, La possession en droit foncier camerounais, Rapport camerounais au Colloque de l'Association Henri Capitant, Yaoundé, 1990, pp. 439 et s. 
pour l'attester, surtout chez les autochtones ${ }^{6}$ qui entendent souvent défendre les "terres de leurs ancêtres" par tous les moyens, au besoin par la violence physique. Le sentiment d'être propriétaire n'existe pas seulement chez les autochtones. On le retrouve même chez les allogènes occupant des terres du domaine national aux alentours des centres urbains. A son arrivée, l'occupant se contente de mettre sur pied une simple baraque en matières provisoires. Quelques temps après, la baraque est remplacée par une construction en matériaux définitifs, ce qui ne laisse plus de doute sur l'intention d'y demeurer à perpétuité, avec le sentiment d'être devenu propriétaire des lieux. La vente des parcelles aux nouveaux arrivants par les premiers occupants est encore plus révélatrice de la conviction d'être devenu propriétaire qui anime ces occupants, qui sont en n'en plus douter, des possesseurs. On retrouve bel et bien dans leur comportement, le corpus et l'animus.

La possession est un fait. Mais sous d'autres aspects, elle se présente aussi comme un droit. D'abord parce que la possession est un concept élaboré par le droit objectif qui en fixe les composantes; ensuite parce que du simple fait possessoire, on peut arriver à acquérir un droit. C'est ce qui arrive lorsqu'il y a prescription acquisition ou usucapion ; La possession confère même des droits, notamment le droit à la protection à travers les actions possessoires. Tout ceci apparaît sur un plan tout à fait général. Il est plus intéressant de s'interroger sur ce que le droit positif camerounais réserve à ces possesseurs des terres du domaine national, à cette grande majorité de la population occupant ou exploitant des espaces du domaine national pour les besoins de survie le plus souvent. Elle y vit paisiblement, au vu et au su des pouvoirs publics.

Dans un pays essentiellement agricole comme le Cameroun, le terre représente un enjeu économique certain. La terre du domaine national qui n'appartient encore à personne est convoitée de toute part. Les éleveurs sont à la recherche de meilleurs pâturages, les cultivateurs à la recherche des terres fertiles, des investisseurs nationaux et étrangers veulent s'y installer... Et l'Etat n'est pas du reste: il a aussi besoin d'espace pour mieux jouer son rôle de promoteur du développement, et même parfois pour assurer sa propre survie.

Les premiers occupants des terres du domaine national, qui n'ont pas pensé à se protéger efficacement à l'aide d'un titre juridique sont parfois victimes d'agressions" ${ }^{7}$ de la part des nouveaux prétendants à la terre. Les nouveaux venus peuvent-ils se prévaloir du fait que les premiers occupants n'ont pas de titre officiel pour chercher à les évincer ? Le droit positif

La notion sociologique d'"autochtone" a été juridicisée par la constitution camerounaise du 18 janvier 1996. Voir Prosper Nkou Mvondo: Le régime foncier face à l'exigence constitutionnelle de préservation des droits des populations autochtones, Verfassung und Recht in Übersee, 31 (1998), p. 343.

7

Agressions au sens propre et au sens figuré. 
camerounais ${ }^{8}$ a-t-il prévu une protection juridique de ces occupants sans titre du domaine national ? La présente étude a pour ambition de répondre à ces questions. Elle montre qu'en tant que possesseur, l'occupant sans titre d'une parcelle du domaine national n'est pas sans protection. Toutefois, s'il est en droit de résister lorsque "sa" parcelle est convoitée par un particulier (I), il doit par contre s'incliner lorsque ladite parcelle est sollicitée par l'Etat (II).

\section{L'occupant sans titre face a un particulier}

L'occupant sans titre d'une parcelle du domaine national est juridiquement protégé contre tout trouble en fait provenant d'un particulier (A); il est aussi protégé contre toute tentative d'éviction en droit dont il pourrait être victime (B).

\section{A. La protection contre un éventuel trouble en fait}

Contre les atteintes de fait, l'occupant d'une parcelle du domaine national a la possibilité de s'adresser à l'autorité administrative (1), ou à l'autorité judiciaire (2).

\section{La saisine de l'autorité administrative: Le rôle protecteur des commissions consultatives}

Instituées par la Réforme de 1974 , les Commissions consultatives ${ }^{9}$ n'ont pas seulement pour mission de régler les litiges lors d'une procédure d'immatriculation. Leur compétence s'étend aussi à tout litige foncier sur les dépendances du domaine national occupées. Il est de pratique au Cameroun que, lorsqu'un litige oppose deux particuliers, ceux-ci s'adressent à une autorité administrative, dans un cadre purement informel. L'autorité saisie s'érige alors en juge pour trancher un litige qui relève normalement de la compétence des juridictions ${ }^{10}$. L'intervention de l'autorité administrative en matière foncière ne s'inscrit pas dans

Il ne s'agit ici que l'étude du droit foncier moderne tel qu'il ressort de la Réforme de 1974. Une réforme qui semble avoir mis entre parenthèses le droit foncier coutumier. Pourtant dans le système juridique parallèle à celui de l'Etat, le droit coutumier sur les terres est encore observé.

Les Commissions consultatives ont été créées par la réforme foncière de 1974 (Article 16 al. 2 de $l^{\prime}$ Ordonnance $\mathrm{n}^{\circ} 74 / 1 /$ du 6 juillet 1974). Participant à la gestion des terres du domaine national, les commissions consultatives sont "présidées par l'autorité administrative et comprennent obligatoirement les représentants de l'autorité traditionnelle". Elles ont été organisées par le Décret $n^{\circ}$

10 76/166/ du 27 avril 1976 (articles 12 et suivants).

Voir Prosper Nkou Mvondo, Le dualisme juridique en Afrique noire francophone, du droit privé formel au droit privé informel, Thèse, Droit, Strasbourg, 1995. 
ce cadre. S'agissant des litiges fonciers sur une dépendance du domaine national, le texte foncier permet bel et bien à l'autorité administrative de trancher officiellement des litiges. Elle agit pour cela par le biais de la commission consultative qui se réunit en principe au moins une fois par trimestre sur convocation de son président, qui n'est autre que le Préfet ou le Sous-préfet. C'est en bon droit qu'un occupant ou exploitant sans titre peut saisir l'autorité administrative, lorsqu'il est troublé dans sa possession, ou lorsqu'un tiers tente de l'évincer en fait.

Une étude menée à Ngaoundéré montre que la commission consultative joue un rôle important dans la protection des premiers occupants, aussi sans titre soient-ils.

Affaire Ahmadou Hayatou Bobbo c/ Bouba Adji (14 octobre 1988) ${ }^{11}$

Ahmadou Hayatou Bobbo se plaint de l'occupation illégitime de son terrain par Bouba Adji: "Mes parents exploitent ce terrain il y de cela 110 ans" dit-il devant la commission. Tous les témoins présents approuvent sa version des faits. Quant à Bouba Adji, il prétend que le même terrain lui a été attribué par le Lamido. Une version des faits que les témoins contestent. La décision de la commission est nette: Bouba Adji doit libérer le terrain au profit du premier occupant Ahmadou Hayatou.

La commission reconnaît à l'occupant sans titre un droit d'usage et de jouissance, absolu et exclusif.

\section{Affaire Mlle Memvouda Salomé c/ Yampaing Aboubakar (03 mars 1984) ${ }^{12}$}

"La commission, après avoir effectué une descente sur les lieux et écouté les parties intéressées par le litige a autorisé Mlle Salomé à poursuivre ses travaux de construction arrêtés par Mr. Aboubakar (le défendeur) qui proteste parce que, dit-il, la fondation de la case de la plaignante risque un jour de casser ses tuyaux de conduite d'eau. La commission n'a pas voulu prendre en considération la déclaration de Mr. Aboubakar car celui-ci n'avait pas obtenu l'accord préalable de Mlle Salomé avant de faire passer ses canalisations d'eau".

On comprend alors que l'occupant sans titre a des droits. Mais il faut aussi le reconnaître, ces droits sont limités. On verra ainsi que la Commission consultative ne reconnaît pas à l'occupant sans titre le droit de vendre la parcelle qu'il possède sans titre.

11 Procès-verbal (P.V.) de règlement de litige du 14 octobre 1988. Archives du Service provincial des domaines de l'Adamaoua - Ngaoundéré.

12 P.V. de règlement de litige du 03 mars 1984. Archives du Service provincial des domaines de l'Adamaoua - Ngaoundéré. 


\section{L'affaire Damangang, Mamadou et Haoua Inna c/ Njong Nshara ${ }^{13}$}

est assez illustrative à propos. Le père Damangang entreprend de vendre la terre qu'il occupe depuis longtemps à Njong Nshara, ceci contre l'avis de ses enfants. Pour le Souspréfet, Président de la Commission consultative, "cette vente est illégale, car le vendeur n'a aucun papier juridique l'autorisant à vendre cette parcelle du domaine national".

\section{La saisine de l'autorité judiciaire: Le rôle protecteur du juge civil et du juge pénal}

L'occupant sans titre est protégé sur le plan civil contre toute atteinte de fait (a), il a même des recours sur le plan pénal (b).

\section{a) Protection sur le plan civil}

Dès lors qu'on admet que l'occupant sans titre d'une parcelle du domaine national est un possesseur $^{14}$, va-t-il sans dire qu'il bénéficie des actions particulières appelées "actions possessoires", qui lui permettent de se protéger en saisissant le juge civil lorsqu'il est troublé par un autre particulier, et même de recouvrer sa possession s'il a été évincé en fait? La réponse à cette question n'est pas évidente en droit camerounais de la terre où l'on sait que le possesseur ne bénéficie pas de toutes les conséquences juridiques classiques liées au fait possessoire. Par exemple, on verra que le possesseur immobilier au Cameroun ne peut se prévaloir de la prescription acquisitive (l'usucapion) ${ }^{15}$. De même, la possession immobilière a été amputée de ses fonctions probatoires ${ }^{16}$. Il y alors lieu de se demander si elle n'a pas été amputée des actions possessoires classiques.

Le Professeur Minkoa Shé, partant de la thèse savignienne sur le fondement de la protection possessoire, semble admettre l'existence des actions possessoires en droit foncier camerounais. Épousant la pensée de Savigny, ce juriste camerounais estime que l'objet de la protection possessoire est, avant tout, le maintien de l'ordre social. Si la possession n'est pas protégée, un tiers pourrait venir la troubler en se prétendant propriétaire. Or aucune société ne peut supporter que l'on se fasse justice soi-même, ce qui incontestablement trouble l'ordre public. La politique législative, qui est loin d'aller à l'encontre de la paix

Commission Consultative de l'Arrondissement de Ngaoundéré, procès-verbal du 10 mars 1993, Archives du Service Provincial des Domaines, Ngaoundéré. Voir Introduction.

15 Voir à ce propos, A. Minkoa She (voir n. 5). L'auteur plaide toutefois pour la réintégration de l'usucapion dans le régime foncier camerounais. 
publique, ne peut se permettre d'interdire de quelque manière que ce soit, les actions possessoires.

Au lendemain de la Réforme foncière de 1974, certains juges camerounais, saisis d'actions possessoires sur les terres du domaine national, refusaient de statuer, prétextant que seules les Commissions consultatives, agissant dans le cadre des procédures d'immatriculation, étaient habilitées à connaître de tels litiges. Les tribunaux, pour fuir le débat, invitaient plutôt les demandeurs d'actions possessoires à engager une procédure d'immatriculation afin de voir leur litige réglé par les Commissions consultatives. Il a fallu que la Cour suprême "tape du poing sur la table" en décidant qu'"il n'appartient pas aux tribunaux saisis d'un litige d'imposer une procédure d'immatriculation aux parties, tout en refusant de statuer sur le litige" ${ }^{17}$. En d'autres termes, le juge saisi d'une action possessoire doit statuer. On peut donc dire par conséquent que le droit foncier camerounais admet bel et bien des actions possessoires. De toute façon, après l'intervention de la Cour suprême, les juridictions camerounaises n'hésitent plus à recevoir des actions possessoires.

Il peut donc arriver que l'occupant sans titre soit troublé dans sa possession; qu'il soit victime de faits (ou même d'actes) qui sont en contradiction avec sa possession et qui impliquent une contestation de son droit d'occuper ${ }^{18}$. Le trouble qui donne lieu à une action possessoire ne doit pas être accidentel ${ }^{19}$. Il doit s'agir d'un acte volontaire qui doit justement impliquer, de la part de son auteur, une contradiction à la possession. Peu importe que l'auteur ait agi de bonne foi ${ }^{20}$. A chaque fait troublant la possession, le droit attache le bénéfice d'une action particulière. Il peut s'agir de la complainte, de la dénonciation de nouvelle œuvre, de la réintégrante.

La complainte.- L'occupant d'une parcelle du domaine national, en sa qualité de possesseur peut saisir le Tribunal de première instance pour faire condamner et faire cesser toute agression matérielle commise contre sa possession. Celle-ci peut être atteinte, non seulement par des actes matériels commis sur la parcelle occupée, mais aussi par des actes faits par l'auteur du trouble sur son propre fonds, tels que l'ouverture des vues contraires aux

Voir Mbella Mbappe, Conclusion sous l'arrêt Ngankam Justin c/ Wouatou Norbert, C.S. $\mathrm{n}^{\circ} 62 / \mathrm{cc}$ du 6 janvier 1983, Revue Camerounaise de Droit, n³ 31/32, 1986, p. 310 et s.

Civ, 11 janvier 1910, Dalloz Périodique, 1911 (Cet arrêt déclare que le trouble possessoire, c'est "tout fait matériel ou juridique qui, soit directement et par lui même, soit indirectement et par voie de conséquence, constitue et implique une prétention contraire à la possession d'autrui"). Req. 27 février 1914, Dalloz Périodique, 1917, 1, 194. Les juges du fond apprécient souverainement le caractère des faits (Req. 31 février 1876, Dalloz Périodique 1876, 1, 112).

Ainsi, l'écroulement d'un mur sur un fonds voisin ne constitue pas un trouble de la possessionCiv. 24 novembre 1895, Dalloz Périodique 1895, 1, page 247.

20 Civ. $1^{\mathrm{er}}$ mai 1889, Dalloz Périodique, 1890, 1, page 479. Il arrive régulièrement au Cameroun, que le tiers se croit effectivement propriétaire en vertu d'un droit coutumier qu'il brandit. 
prescriptions légales. Il en est de même lorsque des travaux provoquent sur le fonds voisin des écoulements ${ }^{21}$ ou des inondations ${ }^{22}$.

La dénonciation des nouvelles auvres.- On suppose ici des travaux entrepris par un tiers qui, s'ils étaient continués, provoqueraient un trouble dans l'occupation. L'action en dénonciation de nouvelles œuvres a pour objectif de sanctionner ce trouble qui n'est pas actuel, mais simplement éventuel. L'action ne peut être exercée que si les travaux sont en cours d'exécution $^{23}$ et à la condition qu'ils ne causent pas encore de trouble. Sinon, seul la complainte pourrait être exercée. Par ailleurs, l'action suppose que les travaux ont été entrepris sur un fonds autre que celui du demandeur, car autrement, il y aurait un trouble déjà réalisé.

La réintégrante.- L'occupant peut se voir dépossédé violemment par un tiers. Ce qui arrive fréquemment au Cameroun où l'on assiste parfois à des affrontements armés entre des personnes ayant les mêmes prétentions sur une parcelle du domaine national. La réintégrante est offerte au possesseur actuel lorsqu'il est violemment évincé par une agression contre sa personne ou contre ses biens. Saisi, le tribunal de première instance pourra permettre au plaignant dépossédé de "réintégrer" la parcelle litigieuse. L'occupant est ainsi protégé.

\section{b) Protection sur le plan pénal}

L'article 239 du code pénal camerounais punit celui qui, dans les conditions susceptibles de troubler l'ordre public, pénètre sur les terres occupées paisiblement par autrui. On parle alors de "trouble de jouissance". Le droit pénal protège ainsi le possesseur des terres, qu'il soit ou non propriétaire. L'occupant sans titre d'une parcelle du domaine national, possesseur sans titre, est donc en droit de saisir le juge répressif s'il est troublé dans sa possession par un particulier. On lui reconnait ainsi un droit à une occupation paisible.

\section{B. La protection contre une éventuelle éviction en droit}

Avec la réforme de 1974, il est permis à toute personne d'accéder à un droit foncier sur une parcelle du domaine national. On peut ainsi en devenir concessionnaire ou propriétaire. Encore faut-il que la terre convoitée ne soit pas occupée par quelqu'un d'autre, même sans titre. 


\section{L'occupant sans titre face à un candidat à la concession}

La concession est un contrat à titre onéreux entre l'Etat gestionnaire du domaine national et une personne de droit privé. Le contrat consiste en l'octroi de jouissance au profit du concessionnaire qui s'engage à mettre la terre concédée en valeur pendant une période ne pouvant excéder cinq ans. On parle alors de concession provisoire. Si la mise en valeur est satisfaisante, la concession provisoire peut se transformer en concession définitive qui débouche sur un droit de propriété ou un bail de longue durée sur la parcelle concédée ${ }^{24}$.

L'Etat est le seul gestionnaire des terres du domaine national. En cette qualité et en principe, l'Etat peut passer des contrats de concession sur toutes les terres placées sous sa gestion. Seulement, on se rend compte à la lecture du décret $n^{\circ} 76 / 166$ du 27 juillet 1976 que l'Etat ne peut exercer ce pouvoir que sur les terres libres de toute occupation. En effet, pour qu'une terre du domaine national puisse faire l'objet d'un contrat de concession, il faut qu'elle soit non-occupée, non-exploitée, en droit comme en fait. L'Etat ne peut donc pas donner en concession une terre occupée, même sans titre. On comprend alors que le possesseur, occupant sans titre, ne saurait être inquiété par un éventuel candidat à la concession.

La protection de l'occupant sans titre peut être mise en œuvre dans le cadre de la procédure menée pour l'obtention d'une concession, plus précisément au moment de l'instruction de la demande. La Commission consultative saisie obligatoirement dans ce cadre se doit de descendre sur le terrain afin de se rendre compte que la parcelle sollicitée par le candidat à la concession est bien libre de toute occupation. Si le contraire est observé, c'est-à-dire que si l'on trouve sur le terrain un tiers occupant déjà les lieux, la commission émet un avis défavorable à la demande (article 14). L'occupant victime d'une concession accordée en violation des textes a la possibilité d'attaquer l'acte administratif attributif par voies de contentieux administratif.

\section{L'occupant face à un candidat à l'immatriculation}

L'accession à la propriété d'une parcelle du domaine national passe par une procédure d'immatriculation. Il peut arriver qu'une parcelle occupée sans titre soit sollicitée par un tiers qui entend l'immatriculer à son profit. Peut-on se prévaloir du fait que celui que l'on trouve sur la parcelle sollicitée est un occupant sans titre pour l'évincer? 
La procédure d'immatriculation commence par une demande que le postulant adresse à l'administration qui à son tour en informe le public. Grâce à cette publicité, l'occupant sans titre informé, pourra faire observer à l'administration qu'il est l'occupant effectif de la parcelle sollicitée par la demande dont publicité est faite. Le texte foncier lui permet en effet de faire opposition ${ }^{25}$. Son opposition sera justifiée, car, seul celui qui occupe ou exploite effectivement la terre peut engager une procédure d'obtention du titre foncier. Celui-là qui a entrepris d'immatriculer une parcelle qu'il n'occupe ou n'exploite pas n'est alors en réalité qu'un usurpateur au regard de la loi foncière. Quoi qu'il en soit, l'opposition est examinée par la Commission consultative qui intervient forcément dans la procédure d'immatriculation. Celle-ci a pour mission, entre autres, de descendre sur le terrain constater que la parcelle sollicitée a été mise en valeur par le requérant, comme l'exige la loi.

Présent le jour du constat, l'occupant sans titre va contester la mise en valeur de la terre par celui-là qui entend l'évincer abusivement. Un litige peut ainsi naître entre les deux parties. Le rôle de la Commission consultative est justement de régler ce genre de difficulté sur le terrain. Et s'il est établi que celui-là qui veut immatriculer n'est pas possesseur, il va sans dire que la Commission tranchera en faveur du véritable occupant, aussi sans titre soit-il.

L'opposition faite par le possesseur peut ne pas avoir été réglée le jour du constat. Il peut aussi arriver que l'occupant ne se rende compte que sa possession est en péril qu'après le passage de la commission consultative. Dans ces deux cas, l'occupant n'est pas pour autant désarmé. Les oppositions sont toujours recevables, même après le passage de la Commission. L'occupant a la possibilité de saisir le chef de service provincial des domaines aux fins de bloquer la procédure d'immatriculation engagée. Celle-ci ne poursuivra son cours que s'il y a mainlevée de l'opposition. A défaut de mainlevée, l'opposition est transmise au ministre chargé des domaines après l'expiration d'un certain délai. Avant de trancher le litige, le ministre saisit pour avis, la commission consultative. Il peut soit rejeter la demande d'immatriculation, consacrant ainsi le possesseur dans son occupation sans titre, soit autoriser l'immatriculation sollicitée. La décision du ministre qui lèse les intérêts du possesseur peut être attaquée par les voies du contentieux administratif.

On comprend à travers ces développements que l'occupant sans titre a des droits. Mais seulement, la défense de ces droits n'est vraiment efficace que lorsqu'elle est faite avant l'aboutissement de la procédure d'immatriculation. L'occupant sans titre qui a laissé la procédure aller jusqu'à son terme, perdra la parcelle dont il a la possession si un titre la réquisition d'immatriculation, et jusqu'à l'expiration d'un délai de 30 jours à compter de la publication au Journal officiel de l'avis de clôture de bornage" (article 16, décret 76/165 du 27 avril 1976). 
foncier a fini par être délivré au requérant, aussi usurpateur soit-il. En effet, le titre foncier une fois établi est définitif, inattaquable et intangible ${ }^{26}$.

On a pourtant observé dans la pratique que ce n'est qu'après l'établissement du titre foncier que l'occupant sans titre se rend le plus souvent compte qu'une procédure d'immatriculation a été menée à son insu et à ses dépens. Il se "réveille" alors et tente d'invalider le titre foncier en question. S'agissant du sort qui lui est alors réservé, il faut envisager deux hypothèses:

$1^{\circ}$ - L'occupant lésé établit qu'il n'y a pas eu de publication de la demande d'immatriculation comme l'exige le texte foncier; ou alors que la Commission consultative n'est jamais passée sur le terrain pour constater la prétendue mise en valeur du requérant à l'immatriculation. Pourtant, dans le dossier que tient l'administration, toutes les pièces relatives à ces opérations s'y trouvent. Il s'agit là d'un cas de fraude. Le titre foncier a été délivré sur la base de fausses pièces. Dans ce cas, l'occupant sans titre peut saisir le ministre chargé des domaines. Le titre foncier frauduleusement délivré sera purement et simplement retiré (art. 2, al. 3 du Décret $n^{\circ} 76 / 165$ du 26 avril 1976) ${ }^{27}$. La décision du ministre est susceptible de recours devant les juridictions administratives.

$2^{\circ}$ - Il peut aussi arriver que la procédure ait été normalement menée. Mais, il est établi que le candidat à l'immatriculation a usé de manoeuvrés, de tromperie, d'attitude malhonnête pour surprendre l'administration qui a fini par lui délivrer un titre foncier sur une parcelle occupée par quelqu'un d'autre. Par exemple, il s'est fait passer pour le véritable occupant; et la procédure a suivi son cours normal. Il y a ici dol, et l'occupant sans titre en est victime. Malheureusement pour ce dernier, la parcelle en question ne lui reviendra plus jamais. Le titre foncier ainsi obtenu par dol garde ses caractères définitif, inattaquable et intangible. Il ne reste plus au possesseur lésé qu'une action personnelle en dommages et intérêts devant les juridictions civiles du lieu de situation de l'immeuble.

On peut tout de même observer que l'occupant sans titre ne sera évincé que lorsque sa vigilance aura été trompée. Il a des droits; il lui appartient de les défendre. Ainsi,

Selon l'article 1 alinéa 2 du décret $n^{\circ} 76 / 165$ du 27 avril 1976: "sous réserve des dispositions des articles 2 (alinéa 3) et 24 du présent décret, le titre foncier est inattaquable, intangible, définitif. Il en est de même des actes constatant les autres droits réels attachés à la propriété". La Cour suprême du Cameroun avant 1974 avait déjà affirmé ces caractères du titre foncier: Arrêt $\mathrm{n}^{\circ}$ 44L du 16 janvier 1968, arrêt $\mathrm{n}^{\circ}$ 142L du 4 juin 1968 (cités par A.D. Tjouen, Droits domaniaux et techniques foncières en droit camerounais, page 173 .

27 "Toutefois, le ministre chargé des domaines peut, en cas de faute de l'administration, résultant notamment d'une irrégularité commise au cours de la procédure d'obtention du titre foncier, et au vu des actes authentiques produits, procéder au retrait du titre foncier irrégulièrement délivré", dit cet article. 
l'occupant d'une parcelle du domaine national a des droits qu'ils peut exercer contre des tiers qui tenteraient d'ignorer sa possession, fut-elle sans titre. En face d'autres particuliers, l'occupant sans titre est fortement protégé. Il n'en est pas toujours de même lorsqu'il se retrouve face à l'Etat convoitant la parcelle qu'il occupe sans titre.

\section{L'occupant sans titre face à l'État}

Si l'occupant sans titre d'une parcelle du domaine national peut juridiquement défendre sa possession lorsqu'il se trouve en face d'un particulier, il doit par contre se plier lorsque c'est l'Etat qui sollicite la parcelle qu'il occupe. Sa situation juridique est ici fragile. Toutefois, il faut noter que si celui qui s'est installé avant la réforme de 1974 est ménagé (A), celui qui s'est installé sur le domaine national après la réforme court même des risques s'il refuse de se plier aux sommations de l'administration qui lui demande de"vider les lieux"(B).

\section{A. L'occupant installé avant 1974}

Selon l'article 17 de l'Ordonnance du 6 juillet 1974, toute personne de nationalité camerounaise qui, à la date d'entrée en vigueur de l'ordonnance (5 août 1976), occupe ou exploite paisiblement une parcelle du domaine national, continuera à l'occuper ou l'exploiter. Le texte précise qu'il faut être de nationalité camerounaise.

Des raisons d'ordre social et économique expliquent sans doute cette prise de position du législateur de 1974. Sur le plan social, il aurait été maladroit de demander à des populations installées parfois depuis des siècles de quitter brusquement les terres de leurs ancêtres. Une telle mesure n'aurait aucune chance d'être respectée par lesdites populations. Le législateur a donc tenu à ménager ces occupants de longue date. Sur le plan économique, il aurait été inconséquent de demander aux paysans d'abandonner leurs cultures sous prétexte d'une redistribution des terres. Le développement dont l'Etat se veut garant aura sans doute essuyé des coups.

Quoiqu'il en soit, le législateur n'a pas voulu créer la panique. Pour les occupants installés avant 1974, tout continuera de se passer en fait comme s'il n'y avait pas eu de réforme. Mais seulement en droit, le particulier installé avant 1974 peut se voir dépossédé à tout moment par l'Etat gestionnaire des terres du domaine national, lorsque des besoins d'intérêt général l'exigent. Dans ce cas néanmoins, l'occupant ou l'exploitant, bien que sans titre, a droit à une indemnité. 


\section{La possible dépossession de l'occupant par l'État: Le déguerpissement}

L'Etat dispose de techniques juridiques pour déposséder l'occupant ou l'exploitant sans titre d'une parcelle du domaine national. L'Etat peut procéder par ce que l'on appelle en droit foncier camerounais: le déguerpissement. Lorsque le déguerpissement est fait au profit du domaine public, on parle de "classement"; et lorsque l'opération est faite au profit du domaine privé de l'Etat, on parle "d'incorporation".

\section{a) Le classement}

L'article 18 de l'Ordonnance de 1974, dans sa rédaction de 1977 (art. 1) dispose qu'en vue de la réalisation des opérations d'intérêt public, économique ou social, l'Etat peut classer au domaine public (...), des portions du domaine national. Peut importe, à la lecture du texte, qu'il s'agisse de terres occupées ou de terres libres de toute occupation. On comprend alors qu'il peut suffire d'un acte de l'autorité publique pour que l'occupant sans titre d'une parcelle du domaine national soit dépossédé ${ }^{28}$. Encore que l'acte de l'autorité publique n'a pas besoin d'être expliqué au dépossédé. Ce qui ne veut pour autant pas dire que la décision de l'autorité étatique est arbitraire. La destination de la terre "classée" peut être contrôlée.

La dépossession de l'occupant par le procédé de classement est définitive. Ainsi, même si après classement, la parcelle est reconnue sans utilité compte tenu de son affectation initiale, elle peut être déclassée non au profit de l'ancien occupant sans titre, mais plutôt intégrée par décret au domaine privé de l'Etat ou des autres personnes morales de droit public (article 5, Ordonnance de 1977).

\section{b) L'incorporation}

Selon l'article 18 de l'Ordonnance de 1974, dans sa rédaction du 10 janvier 1977, en vue de la réalisation des opérations d'intérêt public, économique et social, l'Etat peut (...) incorporer dans son domaine privé ou dans celui des autres personnes morales de droit public, des portions du domaine national. Comme pour le classement, peut importe que la parcelle sollicitée soit occupée ou qu'elle soit libre de toute occupation

L'incorporation est une technique plus soucieuse de l'intérêt de l'occupant sans titre du domaine national, tout au moins en ce qui concerne la procédure, même si le résultat est le même qu'en matière de classement: la dépossession. Alors que le décret de classement tombe comme un couperet sur l'occupant sans titre, ce dernier est tout au moins informé au préalable lorsqu'il s'agit de l'incorporation. Il a la possibilité de faire des observations qui, 
même si elles paralysaient difficilement le processus d'incorporation, lui permettraient quand même de mieux défendre ses intérêts, et même de se préparer à quitter les lieux.

La situation juridique de l'occupant sans titre d'une parcelle du domaine national installé avant 1974 n'est pas très éloigné de celle d'un propriétaire qui peut lui aussi être dépossédé par un acte de l'autorité publique. On verra même que la procédure prévue pour déposséder un occupant sans titre renvoit à celle qui est prévu pour l'expropriation pour cause d'utilité publique. En relèvera notamment que, comme l'exproprié, le simple dépossédé sans titre a droit à une indemnité.

\section{Le droit à une indemnité: L'indemnité de déguerpissement}

L'indemnisation de l'occupant dépossédé par l'Etat, se rapproche de celle qui est prévue en matière d'expropriation pour cause d'utilité publique, tout au moins si l'on considère le moment où l'indemnité doit être versée (a); La différence apparaît toutefois par rapport à l'expropriation en ce qui concerne les éléments entrant en ligne de compte dans la fixation du montant à verser au dépossédé (b).

\section{a) Le moment où l'indemnité de déguerpissement doit être versée}

En matière d'expropriation pour cause d'utilité publique, l'article 545 du code civil dispose que l'indemnisation doit être préalable à toute prise en possession de l'immeuble par l'administration. S'agissant du déguerpissement, il est dit à l'article 24 du texte foncier que l'Etat peut, compte tenu de l'urgence du projet, disposer de toutes les dépendances du domaine national, avant le paiement de l'indemnité. Ce qui veut dire a contrario qu'en principe, l'indemnité de déguerpissement doit être payée avant la dépossession de l'occupant sans titre. Ce n'est qu'exceptionnellement, lorsqu'il y a urgence, que le principe sera mis à l'écart.

\section{b) Le montant de l'indemnité de déguerpissement}

L'indemnité d'expropriation comporte selon l'article 9, non seulement la valeur des cultures, celle des constructions et autres aménagements, mais aussi la valeur du terrain. Par contre, l'indemnité de déguerpissement ne correspond qu'à la valeur des cultures, constructions que supportent le terrain. On ne tient pas ici compte de la valeur du terrain nu comme en matière d'expropriation. Question sans doute de rappeler au déguerpi qu'il n'est pas propriétaire de cette parcelle qui fait partie du domaine national. L'occupant sans titre, même si sa situation reste fragile face à l'Etat, a quand même quelques droits à faire valoir. Cette faveur que le législateur a accordé à ceux qui sont installés avant la réforme de 1974, est refusée à ceux qui se sont installés sur la domaine national après 1974. 


\section{B. L'occupant s'étant installé après 1974}

Après la publication du texte de la réforme de 1974, on va assister à l'installation tout azimut des populations sur les terres du domaine national, sous le prétexte de les mettre en valeur et pouvoir ainsi accéder à la propriété. En réalité, cette attitude des populations est liée à un malentendu. La possibilité d'accéder directement à la propriété foncière n'était offerte qu'à ceux qui avaient mis la terre en valeur avant 1974. Ceux qui aimeraient s'installer nouvellement sur une parcelle du domaine national doivent passer forcément par un contrat de concession provisoire qui peut alors déboucher sur l'obtention d'un titre foncier ${ }^{29}$.

Pour lever le malentendu, les pouvoirs publics ont mené une vaste campagne d'information et d'explication du texte foncier aux populations ${ }^{30}$. Si le phénomène des occupations anarchiques des terres du domaine national se poursuit aujourd'hui, il s'agit beaucoup plus d'un problème de résistance au droit officiel. Pour surprenant que cela puisse paraître, ces populations qui envahissent ainsi les terres du domaine national sont rarement inquiétées par les pouvoirs publics qui réagissent difficilement face à ce qui n'est rien d'autre qu'une occupation illégale $^{31}$. Des sommations de quitter sont souvent restées sans suite.

Sur le plan strict du droit, l'article 4 de la loi $n^{\circ} 80 / 22$ du 14 juillet 1980, punit d'une amende de 50000 à 200000 et d'un emprisonnement de deux(2) mois à trois(3) ans ou de l'une de ces deux peines seulement, celui qui, en violation des lois en vigueur, exploite ou se maintient sur une dépendance du domaine national.

En interprétant strictement cette loi pénale ${ }^{32}$, on finit par comprendre que ce qui est interdit par la loi, c'est l'exploitation" sans autorisation des terres du domaine national. L'occupation" simple n'est pas retenue par le texte pénal. Quelle différence y a-t-il entre

Après la publication du texte foncier en 1974, les tribunaux ont été encombrés de litiges. A.D. Tjouen (voir n. 26) écrit: "Il a fallu que les chefs d'unités administratives tiennent des réunions d'informations des administrés pour faire cesser des revendications absurdes de propriété provoquées par le réforme", op.cit., page 88.

31 Cet état des choses est pourtant loin de faire plaisir aux autorités publiques. Au cours de la réunion tenue le 16 avril 1991 relative à l'examen des problèmes posés par l'occupation anarchique du quartier Mabanga à Ngaoundéré, le Maire de cette ville déclare: "C'est une situation assez connue. On observe de l'autre côté de la gare marchandise un quartier dont le développement se fait contre le gré du plan d'urbanisme qui avait été prévu". Ce dernier, selon le procès-verbal exprimait ainsi "sa déception pour ce quartier qu'on observe à première vue, dès qu'on débarque de l'avion et qui déshonore la ville". Archives du Service provincial des domaines. Procès -verbal de ladite réunion.

En effet, la loi pénale est d'interprétation stricte. Voir $P$. Escande, L'interprétation par le juge des règles écrites en matière pénale", Revue des Sciences Criminelles, 1978, page 811. 
"exploiter" et "occuper". Selon le dictionnaire ${ }^{33}$, "exploiter " est l'action de tirer profit d'une chose que l'on fait produire. Il est donc interdit d'utiliser le domaine national à des fins industrielles ou commerciales. Ainsi en est-il de celui qui y fait de l'agriculture intensive, qui livre les produits de la terre dans un grand marché, tout en y tirant de gros revenus; celui-là tombe sous le coup de la loi pénale. Il en sera sans doute de même de celui qui y construit des logements pour les mettre en location. Dans l'un et l'autre cas cités en exemple, il y "exploitation" du domaine national. L'Etat peut alors saisir le juge pénal qui, après condamnation pénale, pourra ordonner le déguerpissement aux frais de l'exploitant délinquant.

Par contre, le particulier qui s'installe sans tirer de gros profit de la terre, qui utilise le sol pour sa subsistance (case d'habitation, culture d'alimentation...), est un simple occupant. De ce seul fait, il n'encourt pas de sanction pénale. Son comportement est toléré en droit. Toutefois, il se doit de quitter les lieux dès la première sommation de l'autorité étatique gestionnaire des terres qu'il occupe sans titre. Si l'occupant ne s'exécute pas, il tombe alors sous le coup de l'article 8 qui punit non seulement ceux qui exploitent, mais aussi ceux qui se maintiennent sur un terrain dont ils ne sont pas propriétaires.

Il faut quand même relever que ce texte pénal n'a peut-être jamais été appliqué alors que l'on observe tous les jours des cas de violation de cette loi foncière. L'Etat, au lieu de sévir, préfère généralement être passif ${ }^{34}$. Des négociations sont même souvent engagées avec les occupants, pour des raisons d'ordre sociologique ou politique. A l'issue des négociations, les occupants acceptent de quitter les lieux à condition que les pouvoirs publics leur trouvent des parcelles de recasement, avec versement d'une indemnité de "déguerpissement" ${ }^{35}$. Pour faire respecter les plans d'urbanisme qui passent généralement par des terres déjà envahies par les populations, les pouvoirs publics s'engagent souvent à aménager et à procéder au lotissement des lieux. Et les bénéficiaires de ces lotissements sont en priorité, aussi curieux que cela puisse paraître, ceux-là qui occupaient illégalement les parcelles ${ }^{36}$.

33

34

Le dictionnaire Larousse .

Le journaliste Camerounais Alex Blérot Momha le relève justement dans un débat télévisé le 17 février 1998. Il s'indigne face à la démission de l'Etat qui a du mal à interdire aux populations de s'installer dans des zones dangereuses (Débat télévisé organisé par la Télévision Camerounaise après la catastrophe de Nsam qui a coûté la vie à plus de 200 personnes).

Dans le débat télévisé cité ci-dessus, le Ministre de l'administration territoriale relève que les sinistrés occupaient illégalement les lieux. Pour faire face à la situation, il envisage plutôt une mesure de déguerpissement et de recasement de ces populations dans des zones viables.

Au cours de la réunion du 16 avril 1991 (déjà citée), les pouvoirs publics se trouvent face à une alternative:" $1^{\circ}$ Soit déguerpir les occupants et récupérer le terrain, pour le lotir en respectant le plan d'urbanisme prévu, ce qui ne manquera pas de soulever des problèmes sociaux ; $2^{\circ}$ soit stopper cette occupation et procéder ensuite à une restructuration dudit terrain". La deuxième solution sera adoptée à l'unanimité des participants, pour des raisons sociales. On pourra faire passer les 
Néanmoins, l'article 8 existe et garde sa force. Il peut être mis en application à tout moment. Il y donc lieu de dire qu'au regard de la loi, l'exploitant sans titre installé après 1974 court un grand risque, alors que le simple occupant vit dans une précarité constante.

\section{Conclusion}

L'occupant sans titre d'une parcelle du domaine national a des droits qui lui sont reconnus par la réforme de 1974. Qui dit droits, dit forcement limites de ceux-ci. Mais dans la pratique, les occupants sans titre bénéficient généralement de la mansuétude de l'Etat qui, pour des raisons d'ordre social, politique, économique..., refuse le plus souvent de faire valoir les droits des uns et des autres. L'observateur des réalités foncières au Cameroun se rendra bien compte que les occupants sans titre bénéficient de plus de "droits" que ce qui leur est accordé par la législation. On a même parfois le sentiment que cette législation n'existe pas; ou alors que l'Etat qui est chargé de l'application du droit a placé celui-ci dans les tiroirs.

Il faut toutefois faire attention. L'Etat sait souvent se comporter comme un monstre. Un monstre qui peut s'endormir ou alors qui fait semblant de s'endormir, qui peut se réveiller à tout moment et taper du poing sur la table pour que la récréation et l'anarchie prennent fin. A ce moment là, chacun retrouve la place qui est sienne, conformément à la législation. C'est peut être à ce moment-là que cet article, qui présente objectivement le droit tel qu'il est écrit, sans se soucier des réalités sur le terrain, pourra être lu avec beaucoup d'intérêt par les protagonistes de la question foncière au Cameroun.

routes, et seules les cases touchées par le tracé seront détruites. Les autres resteront en place. Voir Procès-verbal de ladite réunion. Archives du Service Provincial des domaines de l'Adamaoua, Ngaoundéré. 